DOI: $10.20472 / B M .2021 .9 .1 .004$

\title{
A CROSS-SECTOR INTERNATIONAL STUDY OF REMOTE WORK AND ITS MANAGEMENT IN THE PANDEMIC
}

\author{
BARBARA A. MANKO, JERZY ROSINSKI
}

\begin{abstract}
:
With the Covid-19 pandemic forcing organizations to work remotely, this paper presents a wide-ranging study of factors affecting the transition from physical to online operations, and the management and conduct of business online. A literature review summarizes years of research on remote work, while an international survey done in August 2020 collects input from managers in 158 organizations dealing with pandemic-related lockdowns. Topics include impacts on productivity, employee morale, and management workload. Sampled organizations range from manufacturing and retail firms to schools and universities. Cross-sector analysis allows inquiry into why, for instance, productivity and morale may decline in some places but improve in others.
\end{abstract}

\section{Keywords:}

Remote work, covid-19 pandemic, managing in the pandemic, organizational behavior in the pandemic, managing remote work, managing work-from-home, managing online, online morale, online productivity

JEL Classification: D83, F01, O39

\section{Authors:}

BARBARA A. MANKO, Penn State University, United States, Email: bam43@psu.edu JERZY ROSINSKI, Jagiellonian University, Cracow, Poland, Email: jerzy.rosinski@uj.edu.pl

\section{Citation:}

BARBARA A. MANKO, JERZY ROSINSKI (2021). A Cross-Sector International Study of Remote Work and its Management in the Pandemic. International Journal of Business and Management, Vol. IX(1), pp. 63-77., 10.20472/BM.2021.9.1.004 


\section{Introduction}

The Covid-19 pandemic has driven changes in the management of organizations worldwide, and management research must now keep pace, producing studies that can be useful for the duration of the pandemic and beyond. This paper-brief in form, but broad in scope - is meant to contribute early steps toward a key goal: multifactor, cross-sector comparative analysis of management issues in the movement to online remote operations.

The paper's two main elements are shaped with this goal in mind. A review of the literature concisely summarizes findings to date on the challenges and opportunities of operating remotely versus in-person. It includes selections from the sizable body of research done in these areas before the pandemic, along with recent articles written in the first months of the pandemic, many of which focus on the initial tumult and management responses as shutdowns of physical facilities began sweeping across various nations.

Next, the paper presents results of an original survey conducted by the authors in August of 2020. The survey reached out to managers and professionals in multiple industries and fields, from manufacturing to education, in countries on four continents. The timing of the survey allowed us to gather responses after the first shocks of the shutdowns had passed and longer-term effects were setting in. Further, the survey's broad reach was intended to capture an overview of impacts and responses across diverse environments, thereby allowing a search for both common patterns and comparative differences.

The survey was exploratory in nature and the paper as a whole is a foundation-building effort, not an attempt to answer particular research questions. Although a great deal of research has been done thus far on the management of remote operations, and on the pros and cons of operating in that manner, much of the work addresses specific problems or aspects of the subject, which of course is valuable. But the overall body of knowledge remains fragmented and big-picture analysis is, for the most part, either lacking or inconclusive.

By presenting this paper, we hope to assist other researchers as well as management practitioners in fleshing out and integrating the larger body of knowledge. The paper points to helpful connections that might be made between findings in different industries, and to potential areas for future research. It also demonstrates the need for a holistic understanding of the wide-ranging impacts of the pandemic. For example, our survey revealed (not surprisingly) that some companies have suffered substantial loss of business while others have held steady or even thrived. This, in turn, raises questions as to how the general health and trajectory of an organization may affect the management of remote work-from-home operations. Our research can only begin to explore such questions; the hope is that studies to come will shed further light.

Ideally, practitioners in the months and years ahead will be able merge big-picture insights with lessons learned from more specialized studies. Research of the latter type is now being produced at a rapid pace. A striking example came in June of 2020, just months after the onset of the pandemic, when the Association for the Advancement of 
Computing in Education (AACE) published an 851-page e-book containing 133 brief research articles, all newly written and all aimed at helping teachers in primary and secondary schools navigate the shift to remote instruction (Ferdig et al, eds., 2020).

Our literature review cites an article from that book, along with other academic research and selected articles from major professional publications and media outlets.

\section{A Review of the Literature on Remote Online Work and Its Management, from Pre- Pandemic Times to the Present}

To say that the Covid-19 pandemic accelerated the adoption of remote online work is perhaps an understatement. The magnitude of the shift needs to be grasped. A research team in the United States, drawing upon workforce data from both government and industry sources, wrote: "Before the pandemic, about $15 \%$ of US employees were working from home at least some of the time. During the first half of April, half of US employees were doing all of their work remotely" (Sull et al., 2020). Given that every economy includes many jobs that cannot be done virtually, the April figure is remarkable. It suggests a rapid and near-total movement of workers whose roles consist of knowledge work, information processing or communication.

Also, the pre-pandemic employee figure of $15 \%$ does not reflect the percentage of organizations that were using remote work before the shutdowns. One would expect this number to be higher, as it was possible for many companies to have only a small portion of their staffs working remotely. And in fact, a 2019 survey for the software firm Condeco found $43 \%$ of U.S. companies reporting the adoption of work-from-home policies to some degree. (Condeco, 2019) This pre-pandemic survey covered companies in five other nations as well-France, Germany, the U.K., Singapore, and Australia - and in all five, the prevalence of existing remote-work policies was similar to that of the U.S., with adoption rates varying from $38 \%$ to $45 \%$. Researchers have hypothesized that prior experience with remote work helped many companies to make the wider-scale transition when the pandemic struck, with one author noting that larger companies also had years of experience in using digital technologies to communicate and coordinate across geographically dispersed locations (Taylor, 2020).

Still, linking regional offices to headquarters is not the same as having all staff members work separately from their homes. A fundamental question is whether work-from-home arrangements are beneficial or detrimental, and many find remote work to have benefits for both the employer and employee. Since the shutdowns began, organizations ranging from web-based tech firms to nonprofit charities "have announced policies allowing employees to work from home permanently" (de Prazer, 2020). For employers, the arrangement lowers the costs of providing office space and workplace amenities (Kessler, 2017), while employees enjoy "time and location flexibility and better balance between family and work" (Kwon and Jeon, 2020) - they no longer need to commute to and from work; they can attend to matters at home more easily. Some researchers claim, as did Felstead and Henseke (2017), that the benefits can lead to "increased levels of organisational commitment, enthusiasm and satisfaction" among employees. 
Others, however, point to potential downsides. A 2018 study of digital gig workers in Southeast Asia and Africa found the vast majority of these independent contractors working from their homes, and even before the forced isolation and stresses of the pandemic, many of them reported negative effects such as "social isolation, working unsocial and irregular hours, overwork, sleep deprivation and exhaustion." (Wood et al, 2018) A more recent article, in MIT Sloan Management Review, warned that additional negatives threaten to take hold when entire staffs of full-time employees work from home: "The coronavirus pandemic's office exodus risks diminishing company culture unless leaders take action to support it." As the author described it, culture is a set of shared values, habits, and practices "that guide action and interaction" in the company, and working separately and remotely can erode the social cues and bonds sustaining the culture. (Howard-Grenville, 2020)

And yet another negative was documented by a study of Australian workers forced to work from home by the pandemic: "Many working parents were faced with doing paid work and family care at home simultaneously. Overall, paid work time was slightly lower and unpaid work time was very much higher during lockdown than before it ... For some the pandemic improved satisfaction levels [with work-life balance], but for most they became worse." (Craig and Churchill, 2020)

\section{Effects on productivity and innovation}

All of the above leads to the question: How is productivity affected by working remotely? A pre-pandemic article, which summarized many findings, concluded that impacts on productivity may vary depending on the type of work involved (Useem, 2017). For individual projects and work that requires extended contacts with clients, "the office has little to offer besides interruption"'-and therefore, as long as interruptions at home do not become too much of an issue, working remotely can be more productive (Useem, 2017). On the other hand there is evidence that collaborative work, especially the innovative kind, is more productive when people are physically co-located. One study found that scientific research papers by co-located authors had higher impact, as measured by citations, than papers by authors working together remotely. (Lee et al, 2010). Others have found that software developers collaborated to solve problems in their code more frequently and effectively when they were co-located (Useem, 2017); that remote working can reduce productivity even in seemingly non-collaborative jobs, such call center work (Greene, 2013); and that well-designed offices can stimulate creative interaction in many ways (Waber et al, 2014).

For these reasons, in the years before the pandemic, a number of companies which had been early adopters of work-from-home arrangements began to reverse their policies, bringing employees back into office buildings. The companies included IBM, where the CIO said "agile" work practices could best be achieved by having small teams and their leaders located together (Kessler, 2017). Meanwhile, other firms adopted innovation strategies based explicitly on physical co-location. In 2014, the pharmaceutical firm AstraZeneca opened an incubator for biomedical startup companies in the midst of its 
corporate R\&D facility in Gothenburg, Sweden, so that both the host company and the external startups could benefit from sharing ideas and expertise through close interaction (AstraZeneca, n.d.). Since then, the concept has been expanded to other AstraZeneca R\&D sites, including the Boston BioHub in the U.S. (AstraZeneca, 2020). Another, more widespread example is found in software development. Numerous companies have used the method called pair programming, in which two developers literally work side-by-side on the same task. This method increases the up-front cost of writing code, as it requires two persons to do a job that would normally be done by just one, but the benefits can include a more creative approach to problem-solving and fewer defects that need to be fixed later (Hannay et al, 2009).

Currently, the overall picture regarding in-person vs. remote collaboration is unclear and in flux. As some sources note, much of the research that favors physical co-location was done in the first decade of the 2000s or the early to mid-2010s, before remote collaboration tools became as refined and as widely used as they are now-and even during that earlier time, there were studies showing better results for remote work (Bloom, 2014). Specialized tools for remote interaction now exist, which are more sophisticated than standard videoconferencing and screen-sharing technologies. An expert in pair programming reported that these tools can allow the pair method to be used between remote partners, during the pandemic, in a form that nearly replicates in-person pairing (Prakapchuk, 2020).

However, many work activities are not as structured or as narrowly focused as pair programming. Remote collaboration becomes more challenging in complex exchanges among groups of people, and in cases that call for making judgments about other persons, such as employment interviews and investment decisions-because "we make sense of the world and our interactions through our body language, emotions, and embodied experiences, all of which are much different in a virtual space." (Howard-Grenville, 2020; see also De Rond et al, 2019).

\section{Effects in education}

The transition to remote work has been particularly difficult in many schools and universities. Although there has been a great deal of investment and experience in online education over the years, especially at the tertiary level, many instructors were caught unprepared by the sudden shutdowns of early 2020: They were accustomed to teaching mainly in person and simply tried to use the same methods online, which does not work well (Supiano, 2020). Indeed, even in non-emergency settings prior to the pandemic, Instructors have often struggled with both the design and management of online courses (Sanga, 2019).

Moreover, students as well as instructors must adapt when education becomes virtual. They are isolated from classroom supervision and support, and this adaptation is typically hardest for students in the primary and secondary grade levels, as they tend to be less mature than older students (Sanga, 2019). In a study focused on the shift to online learning in primary and secondary schools, KIekel et al (2020) identified three major 
challenges facing students and teachers alike. One is "lack of student participation and accountability," with reports noting that low-performing students tend to deteriorate further when learning online. Second, there are challenges related to "technology usage," and the third major area of challenge is a wide variety of "personal frustrations" on the part of both teachers and students. (Kiekel et al, 2020).

\section{Possible keys to rapid and sustained adaptation}

Ultimately, the pandemic has forced a series of tests and learning exercises upon managers and organizations. The initial shutdowns tested their ability to transition rapidly to new modes of work, and then the following months became an extended exercise in sustaining performance and improving it where possible. Researchers have stepped in to find and document lessons learned. We close this literature review with a few examples of the findings.

In academic circles, the ability of universities to move quickly to online operations (or not) became an object of management research. A U.S. research paper identified three success factors: distributed decision-making, frequent multi-channel communication, and "servant leadership," which the authors defined as prioritizing the needs of others and emphasizing "empowerment, involvement, and collaboration" (Fernandez and Shaw, 2020).

Similarly, a study of IT services in India found that managers in one large firm quickly learned "the basics of virtual engagement" when the pandemic forced all service personnel to work from their homes. A key step was the formation of "agile, empowered teams" supported by "regular leadership calls keeping everyone aligned." The teams were empowered to communicate and gather information freely throughout the firm while making decisions on how to serve their clients most effectively. (Shankar, 2020)

In Malaysia, a study of employees at major telecommunication companies showed that continued training during the pandemic can positively impact job performance and satisfaction, and may therefore help companies stay competitive in difficult times (Kanapathipillai and Azam, 2020).

In health care, the pandemic greatly accelerated the use of telemedicine, as many patient consultations and diagnostics moved to a remote basis. One case study showed the feasibility of rapidly implementing remote triaging for cancer patients, concluding that such methods "will be a prominent part of the outpatient clinical practice for the near future" (Paleri et al, 2020).

Other examples abound, not all of them formally documented in research papers. Business-media outlets and professional publications have provided ongoing platforms for practitioners and consultants to share their insights on managing in the pandemic. To cite two diverse cases: In the web edition of Fast Company, a Singapore-based venture capitalist explained the methods he used to help the startup companies in his portfolio adjust and prepare for long-term survival. (Lauria, 2020) And the Chronicle of Higher 
Education has offered numerous advice articles about online instruction; see for example Darby (2020).

\section{Research Design: A Broad-Based Survey}

As noted earlier, original research for this paper was aimed at exploring impacts and management responses across a broad range of organizations during the pandemic, with an emphasis on the adoption of remote work. We constructed a 26-question survey with spaces for open-ended comments, organized as follows:

- 5 identifying questions, on topics including the organization's type of business (a choice of 12 categories, such as manufacturing, information technology, retail, etc.); and the respondent's position (choices included C-level or founder/owner, manager, and professional with leadership responsibilities). The final question in this group asked the extent to which operations and customer service were virtual before the shutdowns (ranging from "Entirely or almost entirely online or O2O" to "Physical/in-person with minor or no online/O2O" operations).

- 14 questions about the impact of the pandemic and shutdowns on the organization and the organization's responses. Topics included pre-shutdown planning; impacts on operations, revenue, and staffing levels; and effectiveness of responses. There was also an open-ended question asking people to write in "What more could your organization do to help things run smoothly?"

- Finally, 7 questions on the human impact of the shutdowns. Topics included effects on staff morale and motivation, the respondent's workload as a leader or manager, and lessons learned going forward.

LimeSurvey was chosen as the instrument. The survey was emailed in early August of 2020 to a non-random but diverse list of recipients in multiple regions of the world.

Regions included North America, Central Europe (Poland, Germany, Austria, etc.), South America, and the nation of South Africa. The email list was generated primarily from two sources: former graduate business students of the authors, who had finished their studies and were now working; and contact information provided by former private- and publicsector associates of the authors.

A total of TBD copies of the survey were sent. By the end of August we received 158 responses, from persons located across all geographic regions.

\section{Report and Analysis of Survey Results}

Responses came from a very wide range of industries and sectors: all 12 categories in the "Type of business" checklist were checked by multiple respondents. Only 10 of the 158 (6.33\%) reported working in manufacturing or related businesses, perhaps reflecting the fact that most respondents lived in highly developed regions and relatively few in places that are centers of contract manufacturing. Dominant categories included retail and 
consumer services (about $37 \%$ and $28 \%$ respectively), education or philanthropy (about $16 \%$ ), and software/IT and transportation/logistics (each slightly over 10\%).

As to position, 9.5\% identified as high-level executives or founder/owners. The majority identified as either managers or professionals with leadership responsibilities (each $26.6 \%$ ). A surprising number, $17.7 \%$, checked "Other," and we were unable to determine what this signified. Given the economic impact of the pandemic and the less-thanoptimum employment markets in some countries beforehand, it is possible that some of these respondents were in jobs below the professional/managerial levels, or had recently become unemployed or laid off.

Prior to the pandemic, most of the respondents' organizations-70\%-were operating on a physical/in person basis "with minor or no online/O2O (online-to-offline)" components to their business. The remaining $30 \%$ had either "significant" online components or operated "entirely/almost entirely" online or O2O.

\section{General Impacts, Planning, and Responses}

As to general impacts of the pandemic on people's organizations: $29.8 \%$ of those in the survey reported a "large or total drop" in volume of business, with $42.4 \%$ experiencing "major cuts to staff." Conversely, 17\% saw an "increase in demand and revenue," with $22.8 \%$ "adding staff." These numbers fit our expectations. It is well known that some industries, such as travel and hospitality, were hit severely by the pandemic while other types of business, such as online sales and $\mathrm{O} 2 \mathrm{O}$ delivery of goods, ramped up to meet rising demands.

It appears that anticipating and planning for a possible Covid-19 shutdown was not a priority item for many organizations. A stunning $56 \%$ of respondents said they saw "no planning before actual shutdown." Of the rest, the most common response by far $(27 \%)$ was that planning began "one week or more" (but less than one month) before shutdowns began in their area.

Nonetheless, a majority of respondents gave passing grades or even high grades to their organizations for how well they handled the transition overall. On a 0 to 5 scale, where 0 equaled "business failure" and the following numbers represented a poor-to-excellent range, $14.3 \%$ checked the unfortunate 0 box and another $26 \%$ chose low 1 or 2 scores. $11.7 \%$ gave their organizations a middle-of-the-pack 3 and the largest portion, $48 \%$, rated overall response better than average or excellent. Responses to more specific "rating" questions, which asked how clearly policies were communicated and how well employees were supported overall, essentially followed the same patterns.

Also, an important survey item asked about the impact of Covid-19 itself on the organization.

- About 5\% (8 of the 158) reported "Many infections among staff, families, and/or customers, including serious illnesses and/or deaths." 
- $28.5 \%$ reported "Some infections, with moderate business impact and occasional serious health outcomes."

- $\quad 63.9 \%$ reported "Relatively little impact" on physical health from Covid-19.

This too fit an expected pattern, given a world where the virus has taken a heavy in some places and has done varying degrees of harm in others. After seeing the responses to this question we proceeded with our analysis, knowing that actual effects on health were a major concern for only a portion of companies in the survey-but that concerns about health were probably much more widespread.

\section{Access to Resources, Impacts on Interaction and Performance ... and an Area for Future Research}

The vast majority of respondents $(80 \%)$ said their organizations provided the technologies needed for remote work, to most people if not to all. Only $11.6 \%$ declared technology access "a problem with no good solution in sight." Access to information and reference materials was a different matter, however. Nearly half identified these as problem areas, with $28 \%$ seeing "no good solution" ahead. We believe this is a significant finding. It paints a picture of IT for remote work in which many people have the "T" but lack the "I"- the very information that the technologies are supposed to help them acquire and process.

In another set of findings that seem significant, but not surprising, most respondents judged the results of remote work and interaction to be inferior to in-person interaction. 44\% said meetings and group sessions went "not nearly as well" when done remotely; another 19\% answered "not quite as well." Closely tracking these numbers, interaction with customers was judged to suffer by more than $65 \%$ of respondents-although the "not nearly as well" portion (37.6\%) was somewhat lower-and almost identical numbers turned up among respondents saying that productivity had declined. (Some samples of open-ended comments:

"People take advantage of flexible work schedules - not answer customer calls on time." And "Email response from some employees is very slow.")

At the other end of the spectrum, smaller but still noteworthy numbers of respondents said that working remotely had improved group sessions (15\%), customer interaction $(20.8 \%)$, and productivity (18\%). We have not yet been able to trace out patterns in the responses to other survey questions, by the same individuals, which might help to explain why some organizations saw improvements rather than negative impacts from remote work.

For example, it is difficult to tell whether the type of business conducted by the organization makes a difference. The industry categories listed in the survey were broad and general. It is possible that within the software and IT category, or within "consumer services" or "transportation and logistics," there are sub-sectors that lend themselves well to remote work and interaction. Alternately, it is possible that the culture or management structure of the organization is a deciding factor. This is an area where further research 
could be worthwhile, as it would be useful in future years for managers to have a clear understanding of the factors that can make their business a good or not-so-good candidate for remote work.

In other areas related to performance of work: Distractions from working at home were a significant or at least occasional issue for $46 \%$ of respondents. And, in a key question targeted to difficulties within the company, the survey asked all respondents: "What has been most challenging about working separately and remotely?" Three areas of challenge dominated the responses by a wide margin.

- "Keeping people on task"- $31 \%$ of all respondents

- "Dealing with crises or urgencies"-28\%

- "Making and implementing new plans"'-26\%

"Small-team collaboration" and "inter-unit collaboration" drew only a few scattered responses as the most challenging aspects of remote work.

\section{Human Impacts on Morale/Motivation and Manager Workload}

Among all respondents, a large plurality —about $41 \%$ (65 of 158) — said that employee morale and motivation had become "more of an issue" in their organizations. Here, we were able to trace some possible explanatory factors. Over $75 \%$ of those respondents (49 of 65) were in organizations that had little or no online components to their business before the shutdowns. Therefore one factor might be that the transition was still causing problems and frustrations for many.

Also, 32\% (21 out of 65) of the respondents were in organizations that experienced substantial drops in business. This could be another factor, as it seemingly would be difficult to maintain a high-morale, highly-motivated workforce in a company where people are struggling to adapt to a new mode of work amid worries about lost revenue and fears of resulting job loss. (Typical comments: "I'm worried about my job." "We service only $25 \%$ [as many] customers now.")

Conversely, 24 respondents, about $15 \%$ of the survey total, reported improved morale and motivation. Of those 24 , nearly half-11, equaling $46 \%$-worked in organizations that enjoyed increasing demand and revenue. (Some comments from this group: "We are very busy" and "Happy to work from home.")

And an intriguing set of answers came when we asked all respondents how their workloads as managers or leaders had been affected. A striking polarity emerged. 34\% replied "I must do much more to adjust and accommodate" (with another 14\% needing to do "somewhat more") — while 38\% said "My work as a manager/leader is made easier." Only a small minority answered in between. Myriad factors may have contributed to this sharp divergence in leadership workload, and although it would be very helpful to determine exactly how the managing of remote work can become easier, this is another 
case where we suspect that factors not captured by our survey may play a part. We do, however, offer our raw data as a starting point to anyone interested in further research.

\section{Needs Expressed in Open-Ended Responses}

Comments were numerous in response to the open-ended question "What more could your organization do to help things run smoothly?" Two categories stood out, one being the expression of needs for various kinds of technical support and service. These tended to be phrased in general terms, such as "more IT training" or a need for additional tech support staff.

But the greatest number and range of comments on this question, as well as throughout the survey, came from respondents in the education sector. Taken together, they appeared to represent schools and systems experiencing a variety of pressures from teaching remotely. The needs ranged from simple basics ("check with students to make sure they all have access to internet"; "prepare students to log in to school system") to personnel needs ("add more staff to support full-time instructors"), a desire for better internal communication ("stay more connected with professors"), and a desire for better customers: "Most students' expectation is good grades, no work."

Also, one memorable comment implied that many instructors were falling short in their attempts to transition to the online realm: "Have IT department design the classes."

\section{Lessons Learned and Future Prospects}

Another set of interesting responses came when we asked what had been learned, during the shutdown, that could be useful going forward. The most common answers from a list of choices were

- "Seeing who among the staff responded well and who didn't" (33\%)

- "Seeing weaknesses or failure points in the organization" (29.7\%)

Others saw a need for new or better technologies, and 17\% saw "new possibilities" for modes of work or customer service. In a comment, one person reported starting a new software business on the basis of experience gained during the shutdown.

When asked "What will be priorities for your organization when things re-open?", the most common response (51\%) was "Building on new opportunities"-which, presumably, will materialize as economies and societies move through their reopening phases. Nearly all other respondents answered in terms of "recovering" from lost revenue, time, or staffing cuts.

Finally, the emotional impact of the pandemic and shutdowns cannot be ignored. Over $38 \%$ of respondents reported feeling greater anxiety or deeper depression than before. About 26\% replied they felt "fine now, but concerned about the future." And, to close on 
a positive note, we can report that nearly $30 \%$ felt they were doing "better than expected" or even "quite good" and "energized" by the challenges and changes they were facing.

\section{Discussion and Conclusions}

Interpretation of survey results has been included in the sections above. As noted, the results point to interesting opportunities for future research in several areas. Most prominently, deeper levels of comparative analysis would be helpful in identifying:

- factors that contribute to getting better rather than worse performance results in the shift to remote work, and

- factors that influence whether the manager/leader's work becomes more onerous or easier.

The survey that we conducted has several limitations. Length of the survey was kept relatively brief, in order to elicit a good response rate during times that have been busy for many. This in turn made it difficult to get high levels of detail and specificity on a number of matters. Budget and time constraints precluded using interviews as a complement to the survey, and also precluded obtaining very large lists in order to achieve truly global reach of the survey. (Most notably, Asian countries were not represented in numbers that reflect their share of the world's population and economic activities.)

On the other hand, in our view, the survey has virtues. Respondents were sampled across multiple dimensions - industry sectors, sizes of the organizations, geographic locationsand were queried on a diverse range of topics pertaining to pandemic response and remote work. Although the breadth of the survey may have been overly ambitious in some respects, we would argue that it has been, at the least, a useful pilot project in the movement toward multifactor comparative analysis of issues in the management of remote work. If remote work is to be a greater part of our world in the future, this movement must continue.

\section{Acknowledgments}

The work presented here was made possible by many parties. We thank the 158 respondents to our survey, who provided valuable information and frank, insightful commentary. We wish to express deep professional gratitude to the researchers and writers cited in the literature review.

In advance, we thank the scholars chosen to review this paper. Finally we thank our families, and the academic institutions in the U.S. and Poland where we have served as faculty members. Although these institutions provided no direct financial support for the research described here, they have supported us in many other ways and enabled us to fund and perform the work independently. 


\section{References}

AstraZeneca (n.d.). BioVentureHub website.

https://www.azbioventurehub.com/ (accessed 22 October 2020)

AstraZeneca (2020). AstraZeneca Incubator at the Boston BioHub.

https://openinnovation.astrazeneca.com/about-us/astrazeneca-incubator.html (accessed 22 October 2020)

Bloom, N. (2014). To Raise Productivity, Let More Employees Work From Home. Harvard Business Review, January-February 2014. https://hbr.org/2014/01/to-raiseproductivity-let-more-employees-work-from-home (accessed 22 October 2020).

Condeco (2019). The Modern Workplace: People, Places \& Technology. White-paper report. https://www.condecosoftware.com/resources-hub/wpcontent/uploads/sites/8/2019/05/Condeco-workplace-report-2019-Digital-

Copy.pdf?utm_campaign=Global\%20-\%202019\%20-

\%20The\%20Modern\%20Workplace\%202019\&utm_source=MWR-

2019\&utm medium=download-the-report\&submissionGuid=f870ff46-6342-4f67-9b92e324043f8454 (accessed 23 October 2020)

Craig, L. and Churchill, B. (2020). Dual-Earner Parent Couples' Work and Care During COVID-19. Gender, Work, and Organization, 25 June 2020.

https://onlinelibrary.wiley.com/doi/full/10.1111/gwao.12497 (accessed 24 October 2020)

Darby, F. (2020). How to Be a Better Online Teacher. Chronicle of Higher Education, 17 April 2020. https://www.chronicle.com/article/how-to-be-a-better-online-teacher/ (accessed 27 October 2020)

De Prazer, V. (2020). APS CPIG Chair's Report. International Coaching Psychology Review, 15(2), 126.

De Rond, M., Holeman, I. and Howard-Grenville, J. (2019). Sensemaking from the Body: An Enactive Ethnography of Rowing the Amazon. Academy of Management Journal, 62 (6), 1961-1988. https://doi.org/10.5465/amj.2017.1417

Felstead, A., and Henseke, G. (2017). Assessing the growth of remote working and its consequences for effort, well-being and work-life balance. New Technology, Work, and Employment, 32(3), 195-212. doi:10.1111/ntwe.12097

Ferdig, R.E., Baumgartner, E., Hartshorne, R., Kaplan-Rakowski, R. and Mouza, C. (Eds). (2020). Teaching, Technology, and Teacher Education During the COVID-19 Pandemic: Stories from the Field. E-book, Association for the Advancement of Computing in Education (AACE). https://www.learntechlib.org/p/216903/ (accessed 20 October 2020). 
Fernandez, A.A. and Shaw, G.P. (2020). Academic Leadership in a Time of Crisis: The Coronavirus and COVID-19. Journal of Leadership Studies, 14(1), 39-45.

doi: $10.1002 / \mathrm{jls} .21684$

Greene, J. (2013). Does Telecommuting Really Reduce Employee Performance? Cnet, 4 March 2013. https://www.cnet.com/news/does-telecommuting-really-reduce-employeeperformance/ (accessed 17 October 2020)

Hannay, J.E., Dybå, T., Arisholm, E. and Sjøberg, D.I.K. (July 2009). The Effectiveness of Pair Programming: A Meta-Analysis. Information and Software Technology. 51 (7): 1110-1122. doi:10.1016/j.infsof.2009.02.001

Howard-Grenville, J. (2020). How to Sustain Your Organization's Culture When Everyone Is Remote. MIT Sloan Management Review, 24 June 2020.

https://sloanreview.mit.edu/article/how-to-sustain-your-organizations-culture-wheneveryone-is-remote/ (accessed 20 October 2020).

Kanapathipillai, K. and Azam, S.M.F. (2020). The Impact of Employee Training Programs on Job Performance and Job Satisfaction in the Telecommunication Companies in Malaysia. European Journal of Human Resource Management Studies, 4(3). https://oapub.org/soc/index.php/EJHRMS/article/view/857 (accessed 18 October 2020).

Kessler, S. (2017). IBM, Remote-Work Pioneer, Is Calling Thousands of Employees Back to the Office. Quartz, 21 March 2017. https://qz.com/924167/ibm-remote-workpioneer-is-calling-thousands-of-employees-back-to-the-office/ (accessed 23 October 2020)

Kiekel, J., Walters, N.M. and Flores, S. (2020). Teaching with a Non-Traditional Mindset: Lessons Learned from In-Service Teachers. In Teaching, Technology, and Teacher Education During the COVID-19 Pandemic, R.E. Ferdig et al, eds., Association for the Advancement of Computing in Education (AACE), 569-574. https://www.learntechlib.org/p/216903/ (accessed 22 October 2020)

Kwon, M., and Jeon, S. H. (2020). Do Leadership Commitment and PerformanceOriented Culture Matter for Federal Teleworker Satisfaction With Telework Programs? Review of Public Personnel Administration, 40(1), 36-55. https://doiorg.ezaccess.libraries.psu.edu/10.1177/0734371X18776049

Lauria, V. (2020). How My VC Fund Is Helping Our Companies Survive the Pandemic. Fast Company, 10 August 2020. https://www.fastcompany.com/90537958/how-my-vcfund-is-helping-our-companies-survive-the-pandemic (accessed 25 October 2020).

Lee, K., Brownstein, J.S., Mills, R.G. and Kohane, I.S. (2010) Does Collocation Inform the Impact of Collaboration? PLoS ONE, 5(12): e14279.

https://doi.org/10.1371/journal.pone.0014279 
Paleri, V., Hardman, J., Tikka, T., Bradley, P., Pracy, P, and Kerawala, C. (2020). Rapid implementation of an evidence-based remote triaging system for assessment of suspected referrals and patients with head and neck cancer on follow-up after treatment during the COVID-19 pandemic: Model for international collaboration. Head \& Neck, 42(7), 16741680. doi:10.1002/hed.26219

Prakapchuk, R. (2020). Remote pair programming? Oh yes, you can. Atlassian, 2 July 2020. https://www.atlassian.com/blog/technology/remote-pair-programming-toolsprocess (accessed 24 October 2020).

Sanga, M.W. (2019). Doing Instructional Design for Distance Education: An Analysis of Design and Technological Issues in Online Course Management. Quarterly Review of Distance Education, 20(1), 35-45.

Shankar, K. (2020). The Impact of COVID-19 on IT Services Industry-Expected Transformations. British Journal of Management, 31(3), 450-452. doi:10.1111/14678551.12423

Sull, D., Sull, C., and Bersin, J. (2020). Five ways leaders can support remote work. MIT Sloan Management Review, 61(4), 1-10.

Supiano, B. (2020). Why You Shouldn't Try to Replicate Your Classroom Teaching Online. Chronicle of Higher Education, 30 April 2020.

https://www.chronicle.com/newsletter/teaching/2020-04-30 (accessed 19 October 2020)

Taylor, S. (2020). The Modern CFO: Adapting to a Time of Crisis. Strategic Finance, 102(1), 42-47.

Useem, J. (2017). When Working From Home Doesn't Work. The Atlantic, November 2017. https://www.theatlantic.com/magazine/archive/2017/11/when-working-from-homedoesnt-work/540660/ (accessed 22 October 2020).

Waber, B., Magnolfi, J. and Lindsay, G. (2014). Workspaces That Move People. Harvard Business Review, October 2014. https://hbr.org/2014/10/workspaces-that-move-people (accessed 23 October 2020)

Wood, A. J., Graham, M., Lehdonvirta, V. and Hjorth, I. (2018). Good gig, bad gig: Autonomy and algorithmic control in the global gig economy. Work, Employment and Society, 33(1), 56-75. doi:10.1177/0950017018785616 